\title{
A Review of Definitions for Fractional Derivatives and Integral
}

\author{
Edmundo Capelas de Oliveira ${ }^{1}$ and José António Tenreiro Machado \\ ${ }^{1}$ Department of Applied Mathematics, IMECC-UNICAMP, 13083-859 Campinas, SP, Brazil \\ ${ }^{2}$ Institute of Engineering, Polytechnic of Porto, Department of Electrical Engineering, Rua Dr. Antonio Bernardino de Almeida 431, \\ 4200-072 Porto, Portugal
}

Correspondence should be addressed to José António Tenreiro Machado; jtenreiromachado@gmail.com

Received 30 January 2014; Accepted 18 May 2014; Published 10 June 2014

Academic Editor: Riccardo Caponetto

Copyright (c) 2014 E. C. de Oliveira and J. A. Tenreiro Machado. This is an open access article distributed under the Creative Commons Attribution License, which permits unrestricted use, distribution, and reproduction in any medium, provided the original work is properly cited.

This paper presents a review of definitions of fractional order derivatives and integrals that appear in mathematics, physics, and engineering.

\section{Introduction}

In 1695, l'Hôpital sent a letter to Leibniz. In his message, an important question about the order of the derivative emerged: What might be a derivative of order $1 / 2$ ? In a prophetic answer, Leibniz foresees the beginning of the area that nowadays is named fractional calculus (FC). In fact, FC is as old as the traditional calculus proposed independently by Newton and Leibniz [1-4].

In the classical calculus, the derivative has an important geometric interpretation; namely, it is associated with the concept of tangent, in opposition to what occurs in the case of FC. This difference can be seen as a problem for the slow progress of FC up to 1900. After Leibniz, it was Euler (1738) [3] that noticed the problem for a derivative of noninteger order. Fourier (1822) $[3,5]$ suggested an integral representation in order to define the derivative, and his version can be considered the first definition for the derivative of arbitrary (positive) order. Abel (1826) [3,5] solved an integral equation associated with the tautochrone problem, which is considered to be the first application of FC. Liouville $(1832)[3,5]$ suggested a definition based on the formula for differentiating the exponential function. This expression is known as the first Liouville definition. The second definition formulated by Liouville is presented in terms of an integral and is now called the version by Liouville for the integration of noninteger order. After a series of works by Liouville, the most important paper was published by Riemann [6], ten years after his death. We also note that both Liouville and Riemann formulations carry with them the so-called complementary function, a problem to be solved. Grünwald [7] and Letnikov [8], independently, developed an approach to noninteger order derivatives in terms of a convenient convergent series, conversely to the Riemann-Liouville approach, that is given by an integral. Letnikov showed that his definition coincides with the versions formulated by Liouville, for particular values of the order, and by Riemann, under a convenient interpretation of the so-called noninteger order difference. Hadamard (1892) [5] published a paper where the noninteger order derivative of an analytical function must be done in terms of its Taylor series.

After 1900, the FC experiences a fast development and, in an attempt to formulate particular problems, other definitions were proposed. We mention some of them. Weyl [9] introduced a derivative in order to circumvent a problem involving a particular class of functions, the periodic functions. Riesz $[10,11]$ proved the mean value theorem for fractional integrals and introduced another formulation that is associated with the Fourier transform. Marchaud (1927) $[3,5]$ introduced a new definition for noninteger order of derivatives. This definition coincides with the Liouville version for "sufficiently good" functions. Erdélyi-Kober (1940) $[3,5]$ presented a distinct definition for noninteger order of integration that is useful in applications involving integral and differential equations. Caputo (1967) [12] formulated a definition, more restrictive than the Riemann-Liouville 
but more appropriate to discuss problems involving a fractional differential equation with initial conditions [13-21].

Due to the importance of the Caputo version, we will compare this approach with the Riemann-Liouville formulation. The definition as proposed by Caputo inverts the order of integral and derivative operators with the noninteger order derivative of the Riemann-Liouville. We summarize the difference between these two formulations. In the Caputo: first the calculate derivative of integer order and after calculate the integral of noninteger order. In the Riemann-Liouville: first calculate the integral of noninteger order and after calculate the derivative of integer order. It is important to cite that the Caputo derivative is useful to affront problems where initial conditions are done in the function and in the respective derivatives of integer order.

After the first congress at the University of New Haven, in 1974, FC has developed and several applications emerged in many areas of scientific knowledge. As a consequence, distinct approaches to solve problems involving the derivative were proposed and distinct definitions of the fractional derivative are available in the literature. This paper presents in a systematic form the existing formulations of fractional derivatives and integrals. We should mention also that we can have several alternative expressions for the same definition. Therefore, we present only those more representative and we cite particular papers [22-32] and books [33-40] that we believe are the most relevant. Furthermore, the paper does not focus on the pros and cons of each definition and does not address the support of the function that is to be differentiated or integrated.

The paper is organized as follows. Section 2 presents the adopted notation. Sections 3 and 4 list the proposed definitions of fractional derivatives and integrals, respectively. Finally, Section 5 outlines some brief remarks.

\section{Notation}

The following remarks clarify the notation used in the sequel in Sections 3 and 4.

(i) Let $\alpha \in \mathbb{C}: \mathfrak{R}(\alpha) \in(n-1, n], n \in \mathbb{N}$, where $\mathfrak{R}(\cdot)$ denotes the real part of complex number.

(ii) Let $[a, b]$ be a finite interval in $\mathbb{R}, k \in \mathbb{N}, v>0$, and $f(0) \equiv f\left(0^{+}\right)-f\left(0^{-}\right)$.

(iii) The floor function, denoted by $\lfloor\cdot\rfloor$, is defined as $\lfloor x\rfloor=$ $\max \{z \in \mathbb{Z}: z \leq x\}$.

(iv) $[\alpha]$ is the integer part of number $\alpha$ and $\{\alpha\}$ the fractional part, $0 \leq\{\alpha\}<1$, so that $\alpha=[\alpha]+\{\alpha\}$.

(v) $\Delta^{\alpha}\left[f(x)-f\left(x_{0}\right)\right] \simeq \Gamma(1+\alpha) \Delta\left[f(x)-f\left(x_{0}\right)\right]$.

(vi) $\alpha(\cdot, \cdot)$ is the variable fractional order with $0<\alpha(x, t)<$ 1 and $(x, t) \in[a, b] . \alpha(x)$ is a continuous function on $(0,1]$.

(vii) $\mathscr{C}\left(a, z^{+}\right)$is a closed contour, in the complex plane, starting at $\xi=a$, encircling $\xi=z$ once in the positive sense, and returning to $\xi=a \cdot \mu, v \in \mathbb{R} / 0$, with $0<\mu<1$ and $0 \leq v \leq 1$. (viii) Consider $z \in \mathbb{C}$ and $k \in \mathbb{R}$. The so-called $k$-gamma function, denoted by $\Gamma_{k}(z)$, is related to the classical gamma function by means of $\Gamma_{k}(z)=k^{z / k-1} \Gamma(z / k)$.

(ix) The so-called $k$-Pochhammer symbol yields $(z)_{n, k}=$ $\Gamma_{k}(x+n k) / \Gamma_{k}(x)$.

(x) The $k$-fractional Hilfer derivative recovers, as particular cases, the fractional Riemann-Liouville derivative if $v=0$ and $k=1$ and the fractional Caputo derivative if $\nu=1=k[41]$.

\section{Definitions of Fractional Derivatives}

Liouville derivative:

$$
\begin{array}{r}
\mathrm{D}^{\alpha}[f(x)]=\frac{1}{\Gamma(1-\alpha)} \frac{\mathrm{d}}{\mathrm{d} x} \int_{-\infty}^{x}( \\
-\infty-\xi)^{-\alpha} f(\xi) \mathrm{d} \xi, \\
-\infty<x<+\infty .
\end{array}
$$

Liouville left-sided derivative:

$$
\begin{array}{r}
\mathrm{D}_{0^{+}}^{\alpha}[f(x)]=\frac{1}{\Gamma(n-\alpha)} \frac{\mathrm{d}^{n}}{\mathrm{~d} x^{n}} \int_{0}^{x}(x-\xi)^{-\alpha+n-1} f(\xi) \mathrm{d} \xi \\
x>0 .
\end{array}
$$

Liouville right-sided derivative:

$$
\mathrm{D}_{-}^{\alpha}[f(x)]=\frac{(-1)^{n}}{\Gamma(n-\alpha)} \frac{\mathrm{d}^{n}}{\mathrm{~d} x^{n}} \int_{x}^{\infty}(x-\xi)^{-\alpha+n-1} f(\xi) \mathrm{d} \xi
$$

$x<\infty$.

Riemann-Liouville left-sided derivative:

$$
{ }^{\mathrm{RL}} \mathrm{D}_{a^{+}}^{\alpha}[f(x)]=\frac{1}{\Gamma(n-\alpha)} \frac{\mathrm{d}^{n}}{\mathrm{~d} x^{n}} \int_{a}^{x}(x-\xi)^{n-\alpha-1} f(\xi) \mathrm{d} \xi,
$$

$$
x \geq a \text {. }
$$

Riemann-Liouville right-sided derivative:

$$
\begin{array}{r}
{ }^{\mathrm{RL}} \mathrm{D}_{b^{-}}^{\alpha}[f(x)]=\frac{(-1)^{n}}{\Gamma(n-\alpha)} \frac{\mathrm{d}^{n}}{\mathrm{~d} x^{n}} \int_{x}^{b}(\xi-x)^{n-\alpha-1} f(\xi) \mathrm{d} \xi, \\
x \leq b .
\end{array}
$$

Caputo left-sided derivative:

$$
\begin{array}{r}
{ }_{*} \mathrm{D}_{a^{+}}^{\alpha}[f(x)]=\frac{1}{\Gamma(n-\alpha)} \int_{a}^{x}(x-\xi)^{n-\alpha-1} \frac{\mathrm{d}^{n}}{\mathrm{~d} \xi^{n}}[f(\xi)] \mathrm{d} \xi, \\
x \geq a .
\end{array}
$$

Caputo right-sided derivative:

$$
\begin{array}{r}
{ }_{*} \mathrm{D}_{b^{-}}^{\alpha}[f(x)]=\frac{(-1)^{n}}{\Gamma(n-\alpha)} \int_{x}^{b}(\xi-x)^{n-\alpha-1} \frac{\mathrm{d}^{n}}{\mathrm{~d} \xi^{n}}[f(\xi)] \mathrm{d} \xi, \\
x \leq b .
\end{array}
$$


Grünwald-Letnikov left-sided derivative:

$$
\begin{array}{r}
{ }^{\mathrm{GL}} \mathrm{D}_{a^{+}}^{\alpha}[f(x)] \\
=\lim _{h \rightarrow 0} \frac{1}{h^{\alpha}} \sum_{k=0}^{\lfloor n\rfloor}(-1)^{k} \frac{\Gamma(\alpha+1) f(x-k h)}{\Gamma(k+1) \Gamma(\alpha-k+1)}, \\
n h=x-a .
\end{array}
$$

Grünwald-Letnikov right-sided derivative:

$$
\begin{array}{r}
{ }^{\mathrm{GL}} \mathrm{D}_{b^{-}}^{\alpha}[f(x)] \\
=\lim _{h \rightarrow 0} \frac{1}{h^{\alpha}} \sum_{k=0}^{\lfloor n\rfloor}(-1)^{k} \frac{\Gamma(\alpha+1) f(x+k h)}{\Gamma(k+1) \Gamma(\alpha-k+1)}, \\
n h=b-x .
\end{array}
$$

Weyl derivative:

$$
{ }_{x} \mathrm{D}_{\infty}^{\alpha}[f(x)]=\mathrm{D}_{-}^{\alpha}[f(x)]=(-1)^{m}\left(\frac{\mathrm{d}}{\mathrm{d} \xi}\right)^{n}\left[{ }_{x} \mathrm{~W}_{\infty}^{\alpha}[f(x)]\right] .
$$

Marchaud derivative:

$$
\mathrm{D}_{+}^{\alpha}[f(x)]=\frac{\alpha}{\Gamma(1-\alpha)} \int_{-\infty}^{x} \frac{f(x)-f(\xi)}{(x-\xi)^{1+\alpha}} \mathrm{d} \xi .
$$

Marchaud left-sided derivative:

$$
\mathrm{D}_{+}^{\alpha}[f(x)]=\frac{\alpha}{\Gamma(1-\alpha)} \int_{0}^{\infty} \frac{f(x)-f(x-\xi)}{\xi^{1+\alpha}} \mathrm{d} \xi .
$$

Marchaud right-sided derivative:

$$
\mathrm{D}_{-}^{\alpha}[f(x)]=\frac{\alpha}{\Gamma(1-\alpha)} \int_{0}^{\infty} \frac{f(x)-f(x+\xi)}{\xi^{1+\alpha}} \mathrm{d} \xi .
$$

Hadamard derivative [42]:

$$
D_{+}^{\alpha}[f(x)]=\frac{\alpha}{\Gamma(1-\alpha)} \int_{0}^{x} \frac{f(x)-f(\xi)}{[\ln (x / \xi)]^{1+\alpha}} \frac{\mathrm{d} \xi}{\xi} .
$$

Chen left-sided derivative:

$$
D_{c}^{\alpha}[f(x)]=\frac{1}{\Gamma(1-\alpha)} \frac{\mathrm{d}}{\mathrm{d} x} \int_{c}^{x}(x-\xi)^{-\alpha} f(\xi) \mathrm{d} \xi,
$$

Chen right-sided derivative:

$$
x>\mathrm{c} .
$$

$$
\mathrm{D}_{\mathrm{c}}^{\alpha}[f(x)]=-\frac{1}{\Gamma(1-\alpha)} \frac{\mathrm{d}}{\mathrm{d} x} \int_{x}^{c}(\xi-x)^{-\alpha} f(\xi) \mathrm{d} \xi,
$$

Davidson-Essex derivative [15]:

$$
x<\mathrm{c} .
$$

$$
\begin{aligned}
\mathrm{D}_{0}^{\alpha}[f(x)]= & \frac{1}{\Gamma(1-\alpha)} \frac{\mathrm{d}^{n+1-k}}{\mathrm{~d} x^{n+1-k}} \\
& \times \int_{0}^{x}(x-\xi)^{-\alpha} \frac{\mathrm{d}^{k}}{\mathrm{~d} \xi^{k}}[f(\xi)] \mathrm{d} \xi .
\end{aligned}
$$

Coimbra derivative [43-45]:

$$
\begin{aligned}
\mathrm{D}_{0}^{\alpha(x)} & {[f(x)] } \\
= & \frac{1}{\Gamma(1-\alpha(x))} \\
& \times\left\{\int_{0}^{x}(x-\xi)^{-\alpha(x)} \frac{\mathrm{d}}{\mathrm{d} \xi}[f(\xi)] \mathrm{d} \xi+\mathrm{f}(0) x^{-\alpha(x)}\right\} .
\end{aligned}
$$

Canavati derivative:

$$
\begin{array}{r}
{ }_{a} \mathrm{D}_{x}^{\nu}[f(x)]=\frac{1}{\Gamma(1-\mu)} \frac{\mathrm{d}}{\mathrm{d} x} \int_{0}^{x}(x-\xi)^{\mu} \frac{\mathrm{d}^{n}}{\mathrm{~d} \xi^{n}}[f(\xi)] \mathrm{d} \xi, \\
n=\lfloor\nu\rfloor, \quad \mu=n-\nu .
\end{array}
$$

Jumarie derivative, $n=1$ :

$$
\begin{aligned}
\mathrm{D}_{x}^{\alpha}[f(x)]= & \frac{1}{\Gamma(n-\alpha)} \frac{\mathrm{d}^{n}}{\mathrm{~d} x^{n}} \\
& \times \int_{0}^{x}(x-\xi)^{n-\alpha-1}[f(\xi)-f(0)] \mathrm{d} \xi .
\end{aligned}
$$

Riesz derivative:

$$
\begin{aligned}
D_{x}^{\alpha}[f(x)]= & -\frac{1}{2 \cos (\alpha \pi / 2)} \frac{1}{\Gamma(\alpha)} \frac{\mathrm{d}^{n}}{\mathrm{~d} x^{n}} \\
& \cdot\left\{\int_{-\infty}^{x}(x-\xi)^{n-\alpha-1} f(\xi) \mathrm{d} \xi\right. \\
& \left.+\int_{x}^{\infty}(\xi-x)^{n-\alpha-1} f(\xi) \mathrm{d} \xi\right\} .
\end{aligned}
$$

Cossar derivative:

$$
\mathrm{D}_{-}^{\alpha}[f(x)]=-\frac{1}{\Gamma(1-\alpha)} \lim _{N \rightarrow \infty} \frac{\mathrm{d}}{\mathrm{d} x} \int_{x}^{N}(\xi-x)^{-\alpha} f(\xi) \mathrm{d} \xi .
$$

Local fractional Yang derivative [40]:

$$
\left.\mathrm{D}_{-}^{\alpha}[f(x)]\right|_{x=x_{0}}=\lim _{x \rightarrow x_{0}} \frac{\Delta^{\alpha}\left[f(x)-f\left(x_{0}\right)\right]}{\left(x-x_{0}\right)^{\alpha}} .
$$

Left Riemann-Liouville derivative of variable fractional order:

$$
{ }_{a} \mathrm{D}_{x}^{\alpha(\cdot)}[f(x)]=\frac{\mathrm{d}}{\mathrm{d} x} \int_{a}^{x}(x-\xi)^{-\alpha(\xi, x)} f(\xi) \frac{\mathrm{d} \xi}{\Gamma[1-\alpha(\xi, x)]} .
$$

Right Riemann-Liouville derivative of variable fractional order:

$$
{ }_{x} \mathrm{D}_{b}^{\alpha(\cdot,)}[f(x)]=\frac{\mathrm{d}}{\mathrm{d} x} \int_{x}^{b}(\xi-x)^{-\alpha(\xi, x)} f(\xi) \frac{\mathrm{d} \xi}{\Gamma[1-\alpha(\xi, x)]} .
$$

Left Caputo derivative of variable fractional order:

$$
{ }_{a} \mathrm{D}_{x}^{\alpha(\cdot,)}[f(x)]=\int_{a}^{x}(x-\xi)^{-\alpha(\xi, x)} \frac{\mathrm{d}}{\mathrm{d} \xi} f(\xi) \frac{\mathrm{d} \xi}{\Gamma[1-\alpha(\xi, x)]} .
$$


Right Caputo derivative of variable fractional order:

$$
{ }_{x} \mathrm{D}_{b}^{\alpha(\cdot,)}[f(x)]=\int_{x}^{b}(\xi-x)^{-\alpha(\xi, x)} \frac{\mathrm{d}}{\mathrm{d} \xi} f(\xi) \frac{\mathrm{d} \xi}{\Gamma[1-\alpha(\xi, x)]} .
$$

Caputo derivative of variable fractional order:

$$
{ }_{*} \mathrm{D}_{x}^{\alpha(x)}[f(x)]=\frac{1}{\Gamma(1-\alpha(x))} \int_{0}^{x}(x-\xi)^{-\alpha(\xi, x)} \frac{\mathrm{d}}{\mathrm{d} \xi} f(\xi) \mathrm{d} \xi .
$$

Modified Riemann-Liouville fractional derivative:

$$
\mathrm{D}^{\alpha}[f(x)]=\frac{1}{\Gamma(1-\alpha)} \frac{\mathrm{d}}{\mathrm{d} x} \int_{0}^{x}(x-\xi)^{-\alpha}[f(\xi)-f(0)] \mathrm{d} \xi .
$$

Osler fractional derivative [46]:

$$
{ }_{a} \mathrm{D}_{z}^{\alpha} f(z)=\frac{\Gamma(\alpha+1)}{2 \pi i} \int_{\mathscr{C}\left(a, z^{+}\right)} \frac{f(\xi)}{(\xi-z)^{1+\alpha}} \mathrm{d} \xi .
$$

$k$-fractional Hilfer derivative [41]:

$$
{ }^{k} \mathrm{D}^{\mu, \nu} f(x)=\mathrm{I}_{k}^{\nu(1-\mu)} \frac{\mathrm{d}}{\mathrm{d} x} \mathrm{I}_{k}^{(1-\mu)(1-\nu)} f(x) .
$$

\section{Definitions of Fractional Integrals}

Riemann-Liouville left-sided integral:

$$
{ }^{\mathrm{RL}} \mathrm{I}_{a^{+}}^{\alpha}[f(x)]=\frac{1}{\Gamma(\alpha)} \int_{a}^{x}(x-\xi)^{\alpha-1} f(\xi) \mathrm{d} \xi, \quad x \geq a .
$$

Riemann-Liouville right-sided integral:

$$
{ }^{\mathrm{RL}} \mathrm{I}_{b^{-}}^{\alpha}[f(x)]=\frac{1}{\Gamma(\alpha)} \int_{x}^{b}(\xi-x)^{\alpha-1} f(\xi) \mathrm{d} \xi, \quad x \leq b .
$$

Hadamard integral:

$$
\mathrm{I}_{+}^{\alpha}[f(x)]=\frac{1}{\Gamma(\alpha)} \int_{0}^{x} \frac{f(\xi)}{[\ln (\xi / x)]^{1-\alpha}} \cdot \frac{\mathrm{d} \xi}{\xi}, \quad x>0, \alpha>0
$$

Weyl integral:

$$
{ }_{x} \mathrm{~W}_{\infty}^{\alpha}[f(x)]=\frac{1}{\Gamma(\alpha)} \int_{x}^{\infty}(\xi-x)^{\alpha-1} f(\xi) \mathrm{d} \xi .
$$

Chen left-sided integral:

$$
\mathrm{I}_{\mathrm{c}}^{\alpha}[f(x)]=\frac{1}{\Gamma(\alpha)} \int_{c}^{x}(x-\xi)^{\alpha-1} f(\xi) \mathrm{d} \xi, \quad x>c .
$$

Chen right-sided integral:

$$
\mathrm{I}_{c}^{\alpha}[f(x)]=\frac{1}{\Gamma(\alpha)} \int_{x}^{c}(\xi-x)^{\alpha-1} f(\xi) \mathrm{d} \xi, \quad x<c .
$$

Cossar integral [47]

$$
\mathrm{I}_{\mathrm{c}}^{\alpha}[f(x)]=\frac{1}{\Gamma(\alpha)} \int_{c}^{x}(x-\xi)^{\alpha-1} f(\xi) \mathrm{d} \xi, \quad x>c .
$$

Erdélyi (left-sided) integral:

$$
\mathrm{I}_{\sigma, \eta}^{\alpha}[f(x)]=\frac{\sigma x^{-\sigma(\alpha+\eta)}}{\Gamma(\alpha)} \int_{0}^{x}\left(x^{\sigma}-\xi^{\sigma}\right)^{\alpha-1} \xi^{\sigma \eta+\sigma-1} f(\xi) \mathrm{d} \xi .
$$

Erdélyi (right-sided) integral:

$$
\mathrm{I}_{\sigma, \eta}^{\alpha}[f(x)]=\frac{\sigma x^{\sigma \alpha}}{\Gamma(\alpha)} \int_{x}^{\infty}\left(\xi^{\sigma}-x^{\sigma}\right)^{\alpha-1} \xi^{\sigma(1-\alpha-\eta)-1} f(\xi) \mathrm{d} \xi .
$$

Kober (left-sided) integral:

$$
\mathrm{I}_{1, \eta}^{\alpha}[f(x)]=\frac{x^{-\alpha-\eta}}{\Gamma(\alpha)} \int_{0}^{x}(x-\xi)^{\alpha-1} \xi^{\eta} f(\xi) \mathrm{d} \xi .
$$

Kober (right-sided) integral:

$$
\mathrm{I}_{1, \eta}^{\alpha}[f(x)]=\frac{x^{\eta}}{\Gamma(\alpha)} \int_{x}^{\infty}(\xi-x)^{\alpha-1} \xi^{-\alpha-\eta} f(\xi) \mathrm{d} \xi .
$$

Local fractional Yang integral:

$$
{ }_{a} \mathrm{I}_{b}^{\alpha}[f(x)]=\frac{1}{\Gamma(1+\alpha)} \int_{a}^{b} f(\xi)(\mathrm{d} \xi)^{\alpha} .
$$

Left Riemann-Liouville integral of variable fractional order:

$$
a_{x}^{\mathrm{I}_{x}^{\alpha(\cdot,)}}[f(x)]=\int_{a}^{x}(\xi-x)^{\alpha(\xi, x)-1} f(\xi) \frac{\mathrm{d} \xi}{\Gamma[\alpha(\xi, x)]} .
$$

Right Riemann-Liouville integral of variable fractional order:

$$
{ }_{x} \mathrm{I}_{b}^{\alpha(\cdot,)}[f(x)]=\int_{x}^{b}(x-\xi)^{\alpha(\xi, x)-1} f(\xi) \frac{\mathrm{d} \xi}{\Gamma[\alpha(\xi, x)]} .
$$

$k$-fractional Hilfer integral:

$$
\mathrm{I}_{k}^{\alpha} f(x)=\frac{1}{k \Gamma_{k}(\alpha)} \int_{0}^{x}(x-\xi)^{\alpha / k-1} f(\xi) \mathrm{d} \xi
$$

\section{Some Remarks}

Remark 1. If $\mathrm{D}^{\alpha}$ is any fractional derivative, the Miller-Ross sequential derivative of order $k \alpha, k \in \mathbb{Z}$, is given by [3]

$$
\mathscr{D}^{\alpha}=D^{\alpha}, \quad \mathscr{D}^{k \alpha}=D^{\alpha} \mathscr{D}^{(k-1) \alpha} .
$$

Remark 2. Whatever the definition employed, $\mathrm{I}^{0} f(x)=$ $\mathrm{D}^{0} f(x)=f(x)$.

Remark 3. Some authors do not distinguish the definition employed by means of a superscript (GL, RL, C, and L) but use different fonts for the operator instead (D, D, D, D, and $\mathscr{D})$. The particular correspondence between fonts and definitions varies. Very often no indication at all is given, save perhaps in the accompanying text, and the reader is presumed to understand from the context which particular definition is intended. 
Remark 4. In the literature, several alternative notations for operator D may be found:

$$
\begin{aligned}
\mathrm{D}_{a+}^{\alpha} f(x) & =\left(\mathrm{D}_{a+}^{\alpha} f\right)(x)={ }_{a} \mathrm{D}_{x}^{\alpha} f(x)={ }_{a} \mathrm{I}_{x}^{-\alpha} f(x) \\
& =\mathrm{D}_{x-a}^{\alpha} f(x)=\frac{\mathrm{d}^{\alpha} f(x)}{\mathrm{d}(x-a)^{\alpha}}, \\
\mathrm{D}_{b-}^{\alpha} f(x) & =\left(\mathrm{D}_{b-}^{\alpha} f\right)(x)={ }_{x} \mathrm{D}_{b}^{\alpha} f(x)={ }_{x} \mathrm{I}_{b}^{-\alpha} f(x) \\
& =\mathrm{D}_{b-x}^{\alpha} f(x)=\frac{\mathrm{d}^{\alpha} f(x)}{\mathrm{d}(b-x)^{\alpha}} .
\end{aligned}
$$

Only one of the two operators I and D needs to be used, since it is all a matter of changing the sign of $\alpha$. In practice, $D$ is the one more often used.

Remark 5. In the expressions for the right and left Liouville fractional derivatives (2) and (3), respectively, some authors have a slight distinct expression, instead of $0^{+}$just + and at the lower limit $-\infty$.

Remark 6. We can mention the "difference of fractional order," discussed by Bosanquet [48], and the "Ruscheweyh Derivative," presented in [42, 49-51].

Remark 7. The authors' intention is not to discuss pros and cons of the list of definitions of fractional derivatives and integrals in Sections 3 and 4. Having in mind that the reader can find benefits in applying the correct definition for his/her specific research interest, it can be said that the most used definitions are the Riemann-Liouville (e.g., in calculus), the Caputo (e.g., in physics and numerical integration), and the Grünwald-Letnikov (e.g., in signal processing, engineering, and control). The problem of initialization plays an important role in applied sciences and, consequently, various definitions are occasionally adopted within the scope of specific topics, but the overall problem remains to be clarified.

Remark 8. The paper does not focus on particular relations involving explicit parameters, intervals, or constants, associated with the distinct derivatives. For example, we can mention that, for $\mathfrak{R}(\alpha)=0$, with $\alpha \neq 0$, the Liouville fractional derivatives are of purely imaginary order. Also, for $\alpha=n \in \mathbb{N}$, we recover the derivative of integer order. For example, $\mathrm{D}_{+}^{n}[f(x)]=f^{(n)}(x)$ and $\mathrm{D}_{-}^{n}[f(x)]=(-1)^{n} f^{(n)}(x)$.

\section{Conflict of Interests}

The authors declare that there is no conflict of interests regarding the publication of this paper.

\section{References}

[1] M. M. Dzherbashyan and A. B. Nersesian, "About application of some integro-differential operators," Doklady Akademii Nauk (Proceedings of the Russian Academy of Sciences), vol. 121, no. 2, pp. 210-213, 1958.

[2] M. M. Dzherbashyan and A. B. Nersesian, "The criterion of the expansion of the functions to Dirichlet series," Izvestiya
Akademii Nauk Armyanskoi SSR: Seriya Fiziko-Matematicheskih Nauk, vol. 11, no. 5, pp. 85-108, 1958.

[3] K. S. Miller and B. Ross, An Introduction to the Fractional Calculus and Fractional Differential Equations, John Wiley \& Sons, New York, NY, USA, 1993.

[4] K. B. Oldham and J. Spanier, The Fractional Calculus: Theory and Application of Differentiation and Integration to Arbitrary Order, Academic Press, New York, NY, USA, 1974.

[5] J. T. Machado, V. Kiryakova, and F. Mainardi, "Recent history of fractional calculus," Communications in Nonlinear Science and Numerical Simulation, vol. 16, no. 3, pp. 1140-1153, 2011.

[6] B. Riemann, Versuch Einer Allgemeinen Auffassung der Integration und Differentiation. Gesammelte Mathematische Werke und Wissenschaftlicher Nachlass, Teubner, Leipzig, 1876, Dover, New York, NY, USA, 1953.

[7] A. K. Grünwald, "Über "begrenzte" derivationen und deren anwendung," Zeitschrift für Mathematik und Physik, vol. 12, pp. 441-480, 1867.

[8] A. V. Letnikov, "Theory of differentiation with an arbitrary index," Sbornik: Mathematics, vol. 3, pp. 1-66, 1868 (Russian).

[9] H. Weyl, "Bemerkungen zum begriff des differentialquotienten gebrochener ordung vierteljahresschr," Naturforschende Gesellschaft in Zürich, vol. 62, pp. 296-302, 1917.

[10] M. Riesz, "L'intégrale de Riemann-Liouville et le problème de Cauchy," Acta Mathematica, vol. 81, no. 1, pp. 1-222, 1949.

[11] M. Riesz, "L'intégrale de Riemann-Liouville et le problème de Cauchy pour l'équation des ondes," Bulletin de la Société Mathématique de France, vol. 67, pp. 153-170, 1939.

[12] M. Caputo, "Linear models of dissipation whose $q$ is almost frequency independent-ii," Geophysical Journal of the Royal Astronomical Society, vol. 13, no. 5, pp. 529-539, 1967.

[13] R. Figueiredo Camargo, A. O. Chiacchio, and E. Capelas de Oliveira, "Differentiation to fractional orders and the fractional telegraph equation," Journal of Mathematical Physics, vol. 49, no. 3, Article ID 033505, 2008.

[14] R. Caponetto, G. Dongola, L. Fortuna, and I. Petras, Fractional Order Systems: Modeling and Control Applications, World Scientific, Singapore, 2010.

[15] M. Davison and C. Essex, "Fractional differential equations and initial value problems," The Mathematical Scientist, vol. 23, no. 2, pp. 108-116, 1998.

[16] G. Jumarie, "On the solution of the stochastic differential equation of exponential growth driven by fractional Brownian motion," Applied Mathematics Letters, vol. 18, no. 7, pp. 817-826, 2005.

[17] G. Jumarie, "An approach to differential geometry of fractional order via modified Riemann-Liouville derivative," Acta Mathematica Sinica, vol. 28, no. 9, pp. 1741-1768, 2012.

[18] G. Jumarie, "On the derivative chain-rules in fractional calculus via fractional difference and their application to systems modelling," Central European Journal of Physics, vol. 11, no. 6, pp. 617-633, 2013.

[19] A. A. Kilbas, H. M. Srivastava, and J. J. Trujillo, Theory and Applications of Fractional Differential Equations, vol. 204 of North-Holland Mathematics Studies, Elsevier, Amsterdam, The Netherlands, 2006.

[20] C. A. Monje, Y. Chen, B. M. Vinagre, D. Xue, and V. Feliu, Fractional-Order Systems and Controls: Fundamentals and applications, Springer, London, UK, 2010.

[21] I. Podlubny, Fractional Differential Equations: An Introduction to Fractional Derivatives, Fractional Differential Equations, to 
Methods of Their Solution, vol. 198 of Mathematics in Science and Engineering, Academic Press, San Diego, Calif, USA, 1999.

[22] E. C. Grigoletto and E. C. de Oliveira, "Fractional versions of the fundamental theorem of calculus," Applied Mathematics, vol. 4, pp. 23-33, 2013.

[23] G. H. Hardy, "Fractional versions of the fundamental theorem of calculus," The Journal of the London Mathematical Society I, vol. 20, no. 1, pp. 48-57, 2013.

[24] Q. A. Naqvi and M. Abbas, "Complex and higher order fractional curl operator in electromagnetics," Optics Communications, vol. 241, no. 4-6, pp. 349-355, 2004.

[25] Z. M. Odibat and N. T. Shawagfeh, "Generalized Taylor's formula," Applied Mathematics and Computation, vol. 186, no. 1, pp. 286-293, 2007.

[26] E. Capelas de Oliveira and J. Vaz Jr., "Tunneling in fractional quantum mechanics," Journal of Physics A: Mathematical and Theoretical, vol. 44, no. 18, Article ID 185303, 2011.

[27] H. T. C. Pedro, M. H. Kobayashi, J. M. C. Pereira, and C. F. M. Coimbra, "Variable order modeling of diffusive-convective effects on the oscillatory flow past a sphere," Journal of Vibration and Control, vol. 14, no. 9-10, pp. 1659-1672, 2008.

[28] B. Ross, S. G. Samko, and E. R. Love, "Functions that have no first order derivative might have fractional derivatives of all orders less than one," Real Analysis Exchange, vol. 20, no. 2, pp. 140-157, 1994-1995.

[29] V. E. Tarasov, "Fractional vector calculus and fractional Maxwell's equations," Annals of Physics, vol. 323, no. 3, pp. 27562778, 2008.

[30] V. E. Tarasov, Fractional Dynamics: Applications of Fractional Calculus to Dynamics of Particles, Fields and Media, Nonlinear Physical Science, Springer, Heidelberg, Germany, 2011.

[31] J. J. Trujillo, M. Rivero, and B. Bonilla, "On a Riemann-Liouville generalized Taylor's formula," Journal of Mathematical Analysis and Applications, vol. 231, no. 1, pp. 255-265, 1999.

[32] D. Valerio, J. J. Trujillo, M. Rivero, J. T. Machado, and D. Baleanu, "Fractional calculus: a survey of useful formulas," The European Physical Journal Special Topics, vol. 222, no. 8, pp. 1827-1846, 2013.

[33] G. A. Anastassiou, Fractional Differentiation Inequalities, Springer, Dordrecht, The Netherlands, 2009.

[34] D. Baleanu, K. Diethelm, E. Scalas, and J. J. Trujillo, Fractional Calculus: Models and Numerical Methods, vol. 3 of Series on Complexity, Nonlinearity and Chaos, World Scientific, Singapore, 2012.

[35] V. Kiryakova, Generalized Fractional Calculus and Applications, vol. 301, Longman Scientific \& Technical, Harlow, NY, USA, 1994.

[36] F. Mainardi, Fractional Calculus and Waves in Linear Viscoelasticity, Imperial College Press, London, UK, 2010.

[37] A. M. Mathai and H. J. Haubold, Special Functions for Applied Scientists, Springer, New York, NY, USA, 2008.

[38] D. Valerio and J. S. da Costa, An Introduction to Fractional Control, IET, Stevenage, UK, 2012.

[39] X. J. Yang, Local Fractional Functional Analysis and Its Applications, Asian Academic Publisher Limited, Hong Kong, 2011.

[40] X. J. Yang, Advanced Local Fractional Calculus and Its Applications, World Science, New York, NY, USA, 2012.

[41] G. A. Dorrego and R. A. Cerutti, "The $k$-fractional Hilfer derivative," International Journal of Mathematical Analysis, vol. 7, no. 11, pp. 543-550, 2013.
[42] S. G. Samko, A. A. Kilbas, and O. I. Marichev, Fractional Integrals and Derivatives: Theory and Applications, Gordon and Breach Science Publishers, Amsterdam, The Netherlands, 1993.

[43] C. F. M. Coimbra, "Mechanics with variable-order differential operators," Annalen der Physik, vol. 12, no. 11-12, pp. 692-703, 2003.

[44] L. E. S. Ramirez and C. F. M. Coimbra, "On the selection and meaning of variable order operators for dynamic modeling," International Journal of Differential Equations, vol. 2010, Article ID 846107, 16 pages, 2010.

[45] L. E. S. Ramirez and C. F. M. Coimbra, "On the variable order dynamics of the nonlinear wake caused by a sedimenting particle," Physica D: Nonlinear Phenomena, vol. 240, no. 13, pp. 1111-1118, 2011.

[46] T. J. Osler, "Leibniz rule for fractional derivatives generalized and an application to infinite series," SIAM Journal on Applied Mathematics, vol. 18, pp. 658-674, 1970.

[47] J. Cossar, "A theorem on Cesàro summability," Journal of the London Mathematical Society I, vol. 16, pp. 56-68, 1941.

[48] L. S. Bosanquet, "Note on convexity theorems," Journal of the London Mathematical Society I, vol. 18, no. 4, pp. 146-148, 1943.

[49] A. A. Lupas, "Some differential subordinations using Ruscheweyh derivative and Salagean operator," Advances in Difference Equations, vol. 2013, article 150, 2013.

[50] M. M. Meerschaert, J. Mortensen, and S. W. Wheatcraft, "Fractional vector calculus for fractional advection-dispersion," Physica A: Statistical Mechanics and Its Applications, vol. 367, no. 8, pp. 181-190, 2006.

[51] S. Ruscheweyh, "New criteria for univalent functions," Proceedings of the American Mathematical Society, vol. 49, no. 1, pp. 109$115,1975$. 


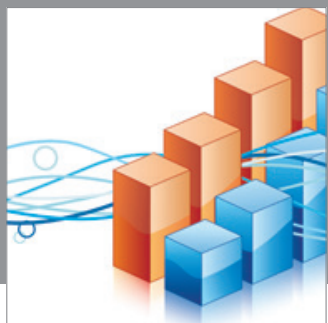

Advances in

Operations Research

mansans

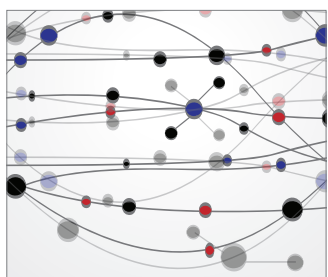

The Scientific World Journal
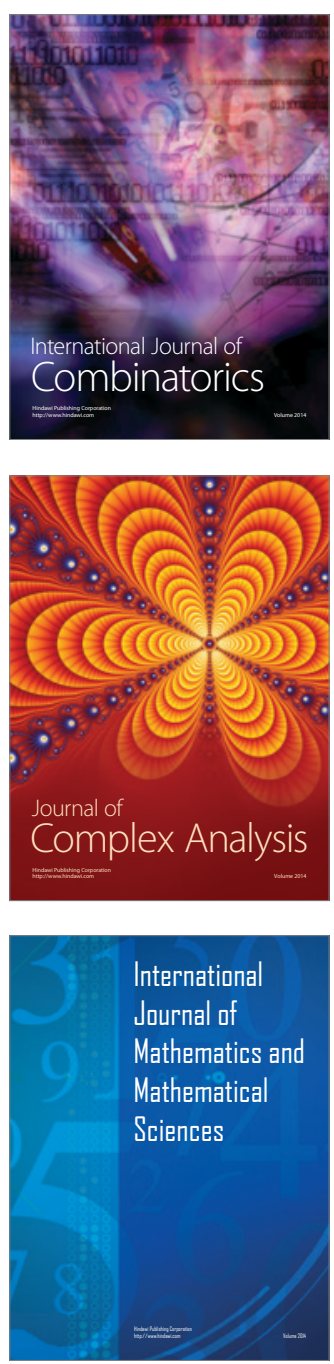
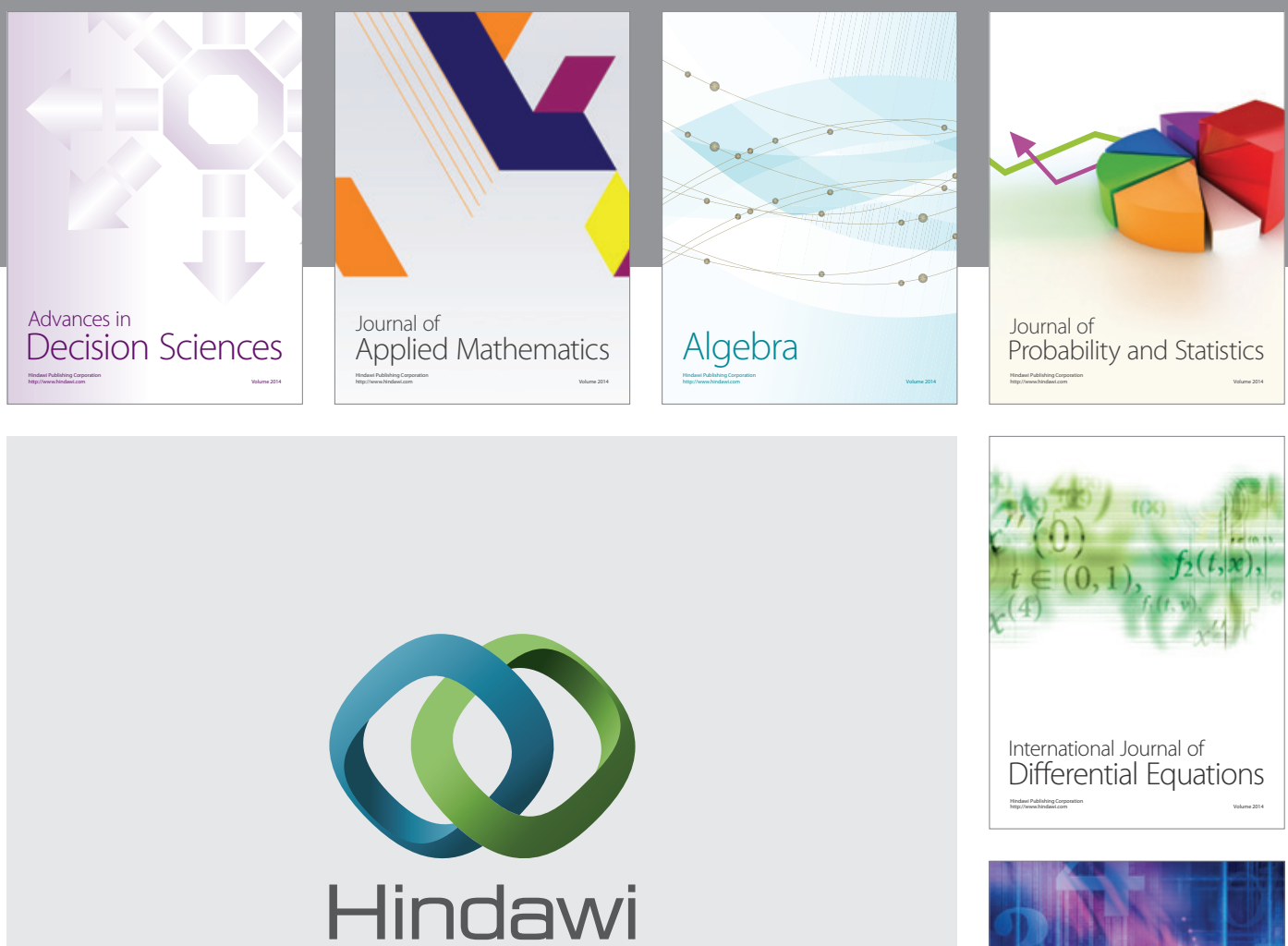

Submit your manuscripts at http://www.hindawi.com
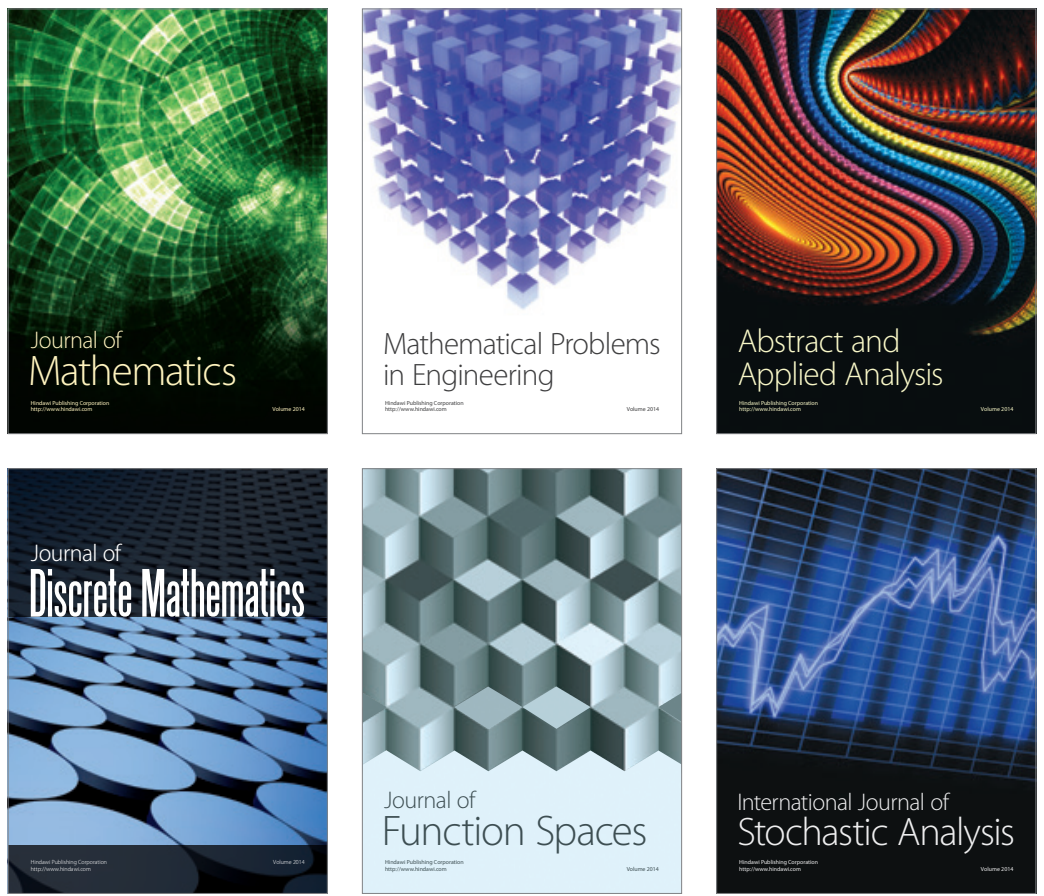

Journal of

Function Spaces

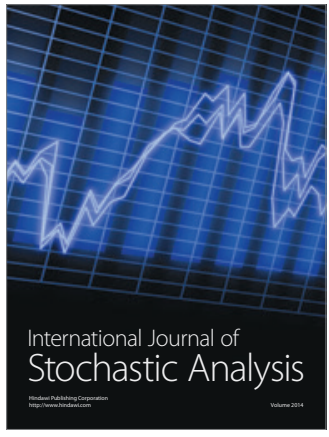

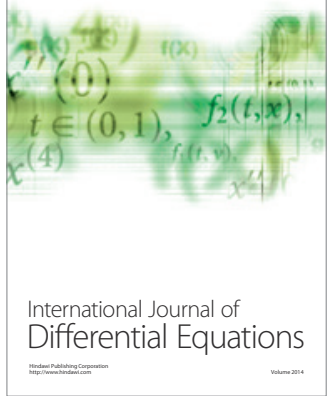
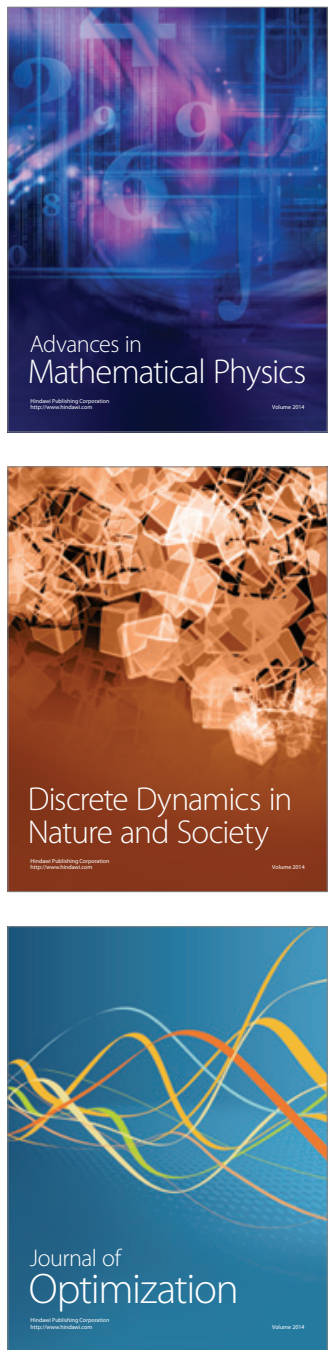